\title{
Antimicrobial Sensitivity Pattern for Urine Isolates in Urinary Tract Infection
}

\author{
Kiran Tauseef Bukhari ${ }^{1}$, Haseeb Tauseef Bukhari², Humaira Zafar ${ }^{1}$, Mudassira Zahid ${ }^{3}$ \\ ${ }^{1}$ Associate Professor, Department of Pathology, Al Nafees Medical College \& Hospital, Islamabad. \\ ${ }^{2}$ PG Trainee FCPS Part-II, Surgical Unit-II, Holy Family Hospital, Rawalpindi \\ ${ }^{3}$ Assistant Professor, Department of Pathology, Al Nafees Medical College \& Hospital, Islamabad
}

\section{AB S TR ACT}

\begin{abstract}
Background: The increased frequency and management of antibiotic resistance pattern in urinary tract infection (UTI) is a challenging task for the clinicians. Therefore, the current study was planned to identify the microbial etiology of UTI and the most suitable antibiotics used. The objective of the study was to assess the frequency of antimicrobial sensitivity pattern in patients with urinary tract infection.

Material and Methods: This cross-sectional study was carried out at Al Sayed Hospital, Kidney Centre, Rawalpindi over a period of seven months i.e. June 30, 2018 to January 30, 2019. A total of 152 patients were enrolled according to the inclusion and exclusion criteria of the study. Urine culture proceedings were done as per latest recommended guidelines of Clinical and Laboratory Standard Institute (CLSI) for UTI.
\end{abstract}

Results: Highest sensitivity was observed for carbapenems (83.5\%), followed by polymyxin B $(72.3 \%)$, phosphonic acid derivatives (65.8\%), aminoglycoside group (65.7\%), extended spectrum penicilin (63.1\%), imidazolidinedione $(59.9 \%)$ and tetracycline $(59.9 \%)$ groups. The least sensitivity was observed for Oxazolidinone (linezolid) (14.5\%), teicoplanin (13.8\%), tigecycline (10.5\%) and firstgeneration cephalosporins (1.3\%).

Conclusions: Carbapenems showed maximum sensitivity for all urine isolates. The second and third options were polymyxin $B$ and phosphonic acid derivatives, respectively.

Key words: Anti-microbial sensitivity, Carbapenems, Phosphonic acid derivatives, Polymyxins, Urinary tract infection, Urine isolates

Authors' Contribution: Correspondence: Article info:

1,2 Conception, synthesis, planning of research Kiran Tauseef Bukhari

Received: January 6, 2019

and manuscript writing Interpretation, discussion, Email:drkirannmc@gmail.com

Accepted: November 23, 2019

${ }^{3,4}$ Active participations in data collection Data analysis.

Cite this article. Bukhari KT, Bukhari HT, ZafarH, Zahid M. Antimicrobial sensitivity pattern for urine isolates in urinary tract infection. J Islamabad Med Dental Coll.2019; 8(4): 186-192.

Doi: $10.35787 / j i m d c . v 8 i 4.325$

Funding Source: Nil

Conflict of Interest: Nil

\section{Introduction}

Urinary tract infection (UTI) is one of the most common bacterial infection in humans. ${ }^{1}$ The infection can be seen in any gender and age group. ${ }^{2}$ UTIs can be further grouped into hospital-acquired and community-acquired infections. ${ }^{3}$ The global statistics report concludes that in adult age group, urinary tract infections (UTIS) are more common in females due to their pelvic anatomy, and diverse physiological changes especially during pregnancy. However, in older age groups the chances of UTI are almost equal in females and males. In cases of males of older age group, the presence of benign prostatic hyperplasia is the top most etiological factor. Complicated UTIs can cause pyelonephritis, or even renal failure and end-stage renal disease. Severe UTI can lead to pre-term delivery and miscarriages in pregnant women. ${ }^{4}$

Gram-positive and Gram-negative bacteria have a role in causing UTI, making it a common bacterial infection. The pathogenesis involves acquisition of genes by microorganisms, which encodes various mechanisms for antibiotic resistance of the organisms. Most important amongst those include gram-negative organisms 
producing extended-spectrum beta-lactamases (ESBLs), AmpC- $\beta$-lactamases, and carbapenemases. ${ }^{5}$

UTI can cause enormous economic burden on the patients from low income communities, and contribute to poor quality of life during the course of the disease. ${ }^{6}$ Irrational and haphazard use of extensive antibiotics is leading to an increase in antimicrobial resistance, especially in the developing countries. 7,8 Hence, appropriate antibiotic use will not only decrease the resistance patterns in the UTI causing organisms butalso help to eliminate the disease quickly, prevent complications and reduce the cost of treatment significantly. ${ }^{9}$

The increased frequency and management of antibiotic resistance in urinary tract infection (UTI) is a challenging task for the clinicians globally. Therefore, the current study was planned to identify the microbial etiology of UTI and the most suitable antibiotics used for treatment. This will be helpful to review the empirical therapy and management protocols of UTI in our set-up.

\section{Material and Methods}

This cross-sectional study was carried out at Al Sayed Hospital, Kidney Centre, Rawalpindi from June 30, 2018 to January 30, 2019. A simple random sampling technique was used to enroll the participants. A sample size of 113 was calculated by taking $12 \%$ frequency of UTI in Pakistan, ${ }^{10}$ however, it was increased to 152 to increase the validity of the study. Both male and female patients of $\geq 18 y e a r s$ of age, having a confirmed diagnosis of UTI based upon findings of urine routine examination $(R / E)$ were included in the study. The presence of more than 10 pus cells in urine R/E were considered significant to label a patient as a suspected case of UTI. ${ }^{11}$ An informed consent was taken from all the enrolled participants to fulfil ethical considerations. The non-willing patients and those with normal urine R/E were excluded from the study.

Three days of urine culture and sensitivity (C/S) were done as per recommended clinical laboratory standard institute (CLSI) guidelines for the year 2017.12 On first day the urine specimens were cultured on CLED agar (Oxoid company). Bacteuric test strips were used for the inoculation of urine culture on CLED agar, so that exact colony counts can be correlated. The CLED agar plates were than incubated at $37^{\circ} \mathrm{C}$ for 24 hours.

On second day the colony counts were correlated with number of pus cells. Gram staining was done to see the bacterial morphology. Bacterial identification was done by using analytical profile index (API-20 E) for biochemical testing. The antimicrobial sensitivity was applied on Mueller Hinton agar.

For beta lactam group, Piperacilin $(100 \mu \mathrm{g})$, Augmentin

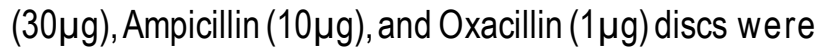
used. Amongst Glycopeptide group, Vancomycin disc $(30 \mu \mathrm{g})$ was used. From Quinolone group, ciprofloxacin $(5 \mu \mathrm{g})$, levofloxacin $(5 \mu \mathrm{g})$, norfloxacin $(10 \mu \mathrm{g})$, ofloxacin $(1 \mu \mathrm{g})$, pipemidic acid $(20 \mu \mathrm{g})$ discs were used. Amongst the Aminoglycoside group, amikacin (30 $\mu \mathrm{g})$ and gentamycin $(10 \mu \mathrm{g})$ discs were used. A doxycycline disc $(30 \mu \mathrm{g})$ was used from Tetracycline group. A $25 \mu \mathrm{g}$ disc of cotrimoxazole (nucleic acid inhibitors) was also used. Regarding cephalosporins, 30 $\mu$ g Cephradine disc was used from first-generation, Cefoxitin $(30 \mu \mathrm{g})$, Cefaclor $(30 \mu \mathrm{g})$, and Cefuroxime $(30 \mu \mathrm{g})$ discs from secondgeneration cephalosporins, and Cefotaxime $(30 \mu \mathrm{g})$, Ceftriaxone $(30 \mu \mathrm{g})$, Cefoperazone $(75 \mu \mathrm{g})$ and Cefixime $(5 \mu \mathrm{g})$ discs were used from third-generation cephalosporins. While from fourth-generation cephalosporins, Cefipime (30 $\mathrm{\mu g}$ ) disc was used. Amongst the carbapenem group, Imipenam disc of $10 \mu \mathrm{g}$ was used. A $30 \mu \mathrm{g}$ Linezolid disc was used from oxazolidinone group. Amongst phosphonic acid derivatives, a fosfomycin disc of $50 \mu \mathrm{g}$ was used. While $300 \mu \mathrm{g}$ Nitrofurantoin disc was used for imidazolidinedione group. More antibiotics used were tigecycline $(15 \mu \mathrm{g})$, teicoplanin $(30 \mu \mathrm{g})$, and polymyxin $(50 \mu \mathrm{g})$, respectively.

On the third day, bacterial confirmation was done by interpreting the results of biochemical tests obtained from API-20E. The antimicrobial zone diameters were measured as per recommended CLSI guidelines, in order to label a drug as sensitive and resistant.

The SPSS Version 16 was used for statistical analysis. Frequencies and percentages were calculated to assess the sensitivity rate, extended spectrum beta-lactamase producing organisms (ESBL), Vancomycin resistant 
Enterococci (VRE) and Methicillin resistant Staphylococcus aureus (MRSA).

\section{Results}

Out of 152 culture positive urine, most prevalent organisms was $E$. coli $(n=81)$ followed by Klebsiella pneumoniae $(\mathrm{n}=25)$ (Table I). Regarding sensitivity pattern, E. coli $(n=77)$, Klebsiella pneumoniae $(n=25)$ and Pseudomonas aeruginosa $(n=7)$ were found maximally sensitive to polymyxin $B$. Enterococcus faecalis showed maximum sensitivity $(\mathrm{n}=16)$ to Carbapenem, Teicoplanin and Glycylcycline (Tigecycline). Enterobacter cloacae revealed highest sensitivity $(n=7)$ to Carbapenem and Phosphonic acid derivatives. Staphylococcus aureus was maximally sensitive $(n=5)$ to Oxazolidinone and Tetracyclines (Table II A and B).

\begin{tabular}{|l|c|c|}
\hline \multicolumn{3}{|c|}{ Table I: Frequency of commonly prevalent organisms in urine } \\
isolates ( $\mathbf{n = 1 5 2 )}$
\end{tabular}

ESBL - Extended-spectrum beta-lactamases; VRE - Vancomycin resistant Enterococci; MRSA - Methicillin resistant Staphylococcus aureus

Considering overall sensitivity of antibiotics for the pathogens of urine culture, highest sensitivity was observed for Carbapenems $(n=127)$, followed by Polymyxin B $(n=110)$, Phosphonic acid derivatives $(n=100)$, Aminoglycoside group $(n=100)$, extended spectrum penicillin ( $n=96)$, Imidazolidinedione $(n=91)$ and Tetracycline groups $(n=91)$. The least sensitivity was observed for first-generation cephalosporins $(n=02)$, Tigecycline ( $n=16)$, Teicoplanin ( $n=21)$ and Oxazolidinone (linezolid) (n=22) (Figure 1)

Amongst all the positive cultures, the highest sensitivity was observed for Carbapenems ( $n=127 ; 83.5 \%)$, Phosphonic acid derivatives $(\mathrm{n}=100 ; 65.8 \%)$ and polymyxin $B(n=110 ; 72.3 \%)$, respectively. This was followed by aminoglycoside group $(n=100 ; 65 \%)$ and extended spectrum penicillin ( $n=96 ; 63.1 \%)$. Next in sequence are the imidazolidinedione and tetracycline groups ( $n=91 ; 59.8 \%$ for each) (Figure 1). The least sensitivity was observed for first-generation Cephalosporins ( $n=02 ; 1.3 \%)$, Tigecycline $(n=16$; 10.5\%), Teicoplanin $(n=21 ; 13.8 \%)$ and Oxazolidinone (Linezolid) ( $n=22 ; 14.4 \%)$, respectively (Figure 1).

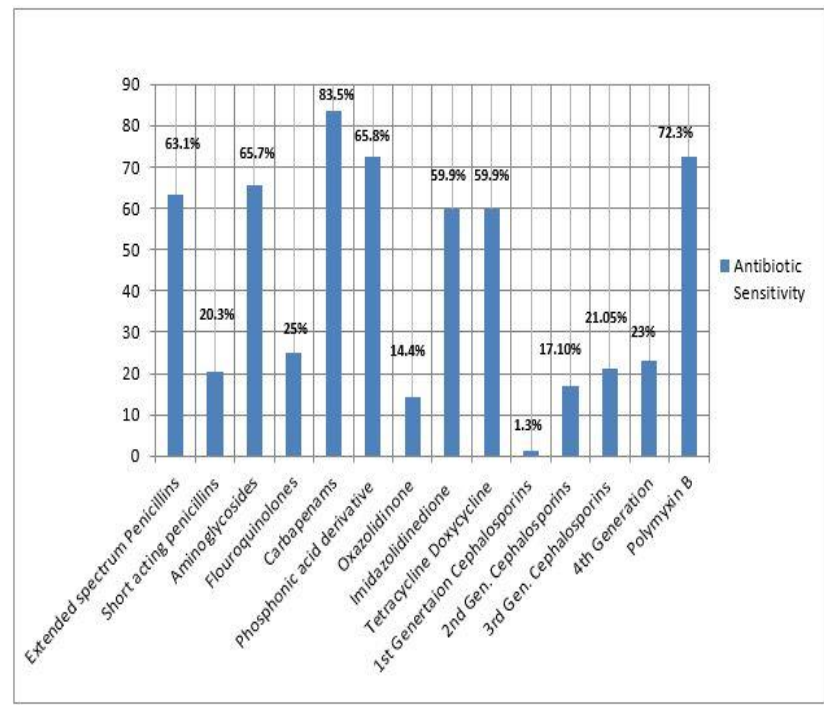

Figure 1: Overall sensitivity of antibiotic groups for pathogens in urine culture $(n=152)$

\section{Discussion}

Urinary tract infections (UTIs) accounts for highly prevalentinfection around the globe. It has been reported that annually 150 million people present with this infection worldwide.13 A study conducted in Denmark on prescription for UTI concluded that amongst all bacterial infections, prevalence of UTI is $89.5 \%$. 


\begin{tabular}{|c|c|c|c|c|c|}
\hline Antibiotics & $\begin{array}{c}\text { Escherichia coli } \\
(n=81) \\
n(\%)\end{array}$ & $\begin{array}{c}\text { Klebsiella } \\
\text { pneumoniae } \\
(n=25) \\
n(\%)\end{array}$ & $\begin{array}{c}\text { Enterococcus } \\
\text { faecalis } \\
(n=18) \\
n(\%)\end{array}$ & $\begin{array}{c}\text { Enterobacter } \\
\text { cloacae } \\
(\mathrm{n}=08) \\
\mathrm{n}(\%)\end{array}$ & $\begin{array}{c}\text { Pseudomonas } \\
\text { aeruginosa } \\
(n=07) \\
n(\%)\end{array}$ \\
\hline Extended spectrum Penicillin & $63(77.7)$ & $04(16)$ & $14(77.8)$ & $01(12.5)$ & $05(71.4)$ \\
\hline Short acting penicillin & $10(12.3)$ & $04(16)$ & $12(66.7)$ & 0 & 0 \\
\hline Aminoglycosides & $71(87.6)$ & $15(60)$ & 0 & $01(12.5)$ & $05(71.4)$ \\
\hline Fluoroquinolones & $26(32)$ & $04(16)$ & 0 & 0 & $05(71.4)$ \\
\hline Sulphonamides & $21(25.9)$ & $3(12)$ & 0 & $01(12.5)$ & 0 \\
\hline Carbapenem & $70(86.4)$ & $23(92)$ & $16(88.9)$ & $07(87.5)$ & $05(71.4)$ \\
\hline Phosphonic acid derivative & $71(87.6)$ & $08(32)$ & $14(77.7)$ & $07(87.5)$ & 0 \\
\hline Oxazolidinone & 0 & 0 & $16(88.9)$ & $01(12.5)$ & 0 \\
\hline Imidazolidinedione & $65(80.2)$ & $10(40)$ & $12(66.6)$ & 0 & 0 \\
\hline Tetracyclines & $61(75.3)$ & $10(40)$ & $15(83.3)$ & 0 & 0 \\
\hline $1^{\text {st } G e n e r a t i o n ~ C e p h a l o s p o r i n s ~}$ & 0 & 0 & $02(11.1)$ & 0 & 0 \\
\hline $2^{\text {nd }}$ Generation Cephalosporins & $22(27.1)$ & 0 & 0 & 0 & 0 \\
\hline $3^{\text {rd }}$ Generation Cephalosporins & $22(27.1)$ & 0 & 0 & 0 & $06(85.7)$ \\
\hline $4^{\text {th }}$ Generation Cephalosporins & $22(27.1)$ & $04(16)$ & 0 & 0 & $05(71.4)$ \\
\hline Polymyxin B & $77(95.06)$ & $25(100)$ & 0 & $01(12.5)$ & $07(100)$ \\
\hline Teicoplanin & 0 & 0 & $16(88.9)$ & 0 & 0 \\
\hline Glycylcycline (Tigecycline) & 0 & 0 & $16(88.9)$ & 0 & 0 \\
\hline
\end{tabular}

\begin{tabular}{|c|c|c|c|c|c|}
\hline \multicolumn{6}{|c|}{ Table II-B: Sensitivity pattern of different microbial isolates from urine cultures ( $n=152)$} \\
\hline Antibiotics & $\begin{array}{c}\text { Staphylococcus } \\
\text { aureus } \\
(\mathrm{n}=06) \\
\mathrm{n}(\%)\end{array}$ & $\begin{array}{c}\text { Morganella } \\
\text { morganii } \\
(n=03) \\
n(\%)\end{array}$ & $\begin{array}{c}\text { Proteus } \\
\text { mirabilis } \\
(\mathrm{n}=02) \\
\mathrm{n}(\%)\end{array}$ & $\begin{array}{c}\text { Burkholderia } \\
\text { cepacia } \\
(\mathrm{n}=01) \\
\mathrm{n}(\%)\end{array}$ & $\begin{array}{c}\text { Serratia } \\
\text { marcescens } \\
(n=01) \\
n(\%)\end{array}$ \\
\hline Extended spectrum Penicillin & $04(66.7)$ & $03(100)$ & $01(50)$ & 0 & $01(100)$ \\
\hline Short acting Penicillin & $04(66.7)$ & 0 & $01(50)$ & 0 & 0 \\
\hline Aminoglycosides & $03(50)$ & 02(66.7) & $02(100)$ & 0 & $01(100)$ \\
\hline Fluoroquinolones & - & 0 & $01(50)$ & $01(100)$ & $01(100)$ \\
\hline Sulphonamides & $03(50)$ & $02(66.7)$ & $01(50)$ & $01(100)$ & $01(100)$ \\
\hline Carbapenem & 0 & $02(66.7)$ & $02(100)$ & $01(100)$ & $01(100)$ \\
\hline Phosphonic acid derivative & 0 & 0 & 0 & 0 & 0 \\
\hline Oxazolidinone & $05(83.3)$ & 0 & 0 & 0 & 0 \\
\hline Imidazolidinedione & $04(66.7)$ & 0 & 0 & 0 & 0 \\
\hline Tetracyclines & $05(83.3)$ & 0 & 0 & 0 & 0 \\
\hline 1st Generation Cephalosporins & 0 & 0 & 0 & 0 & 0 \\
\hline $2^{\text {nd }}$ Generation Cephalosporins & $04(66.7)$ & 0 & 0 & 0 & 0 \\
\hline 3rd Generation Cephalosporins & 0 & 02(66.7) & $01(50)$ & 0 & $01(100)$ \\
\hline $4^{\text {th }}$ Generation Cephalosporins & 0 & $03(100)$ & 0 & 0 & $01(100)$ \\
\hline Polymyxins B & 0 & 0 & 0 & 0 & 0 \\
\hline Teicoplanin & $04(66.7)$ & 0 & 0 & $01(100)$ & 0 \\
\hline Glycylcycline (Tigecycline) & 0 & 0 & 0 & 0 & 0 \\
\hline
\end{tabular}

The appropriate choice for empirical management of UTI is always a challenging task for the clinicians Many national and internationally published studies support the fact that the selection of antibiotic should vary based upon the nature of UTI, acute lower or upper UTI, recurrent
UTI, or a case of complicated UTI. ${ }^{13}$ In the current study results showed that Escherichia coli infection was most common $(55.4 \%)$ followed by Klebsiella pneumoniae $(17.1 \%)$, and Enterococcus faecalis infection (12.3\%). Only few cases were of Burkholderia cepacia and Serratia 
marcescens were reported. These findings are in line with the published data which also showed that Escherichia coli, Klebsiella pneumoniae, Proteus mirabilis, Enterococcus faecalis and Staphylococcus saprophyticus are the main causative organisms for UTI.9,10,14 A Canadian study revealed the same prevalence of uropathogens with the highest frequency seen for gramnegative rods (91.8\%) mainly the Enterobacteriaceae family. ${ }^{15}$ The most common organisms included Escherichia coli (39.7\%), Klebsiella pneumoniae (15.8\%) Pseudomonas aeruginosa (13.8\%), Proteus mirabilis (10.6\%) and Acinetobacter baumannii (5\%). ${ }^{15}$

We also reported the presence of resistant microorganisms like ESBL, VRE and MRSA. ESBL producing Escherichia coli and Klebsiella pneumoniae were seen in $72.8 \%$ and $84 \%$ cases respectively. VRE producing Enterococcus faecalis was seen in $5.5 \%$ cases, while MRSA was seen in $16.6 \%$ cases. This is in agreement with the results of a published study where, Nitrofurantoin and Fosfomycin were the recommended first line treatment options for uncomplicated bacterial cystitis, while the fluoroquinolones and $\beta$-lactams were amongst the second line options. ${ }^{15}$ The results are different from another study conducted in Denmark, where Pivmecillinam hydrochloride (extended-spectrum penicillin) was the first line management option for both lower or upper UTI. ${ }^{13}$ According to published data of various European countries, the identified frequency of ESBLs range from $70-100 \%$, meaning that carbapenems often represent the only active available antibiotics. ${ }^{16}$

Amongst all the positive cultures, the highest sensitivity was observed for carbapenems, followed by Polymyxin B, phosphonic acid derivatives, aminoglycoside group, extended spectrum penicillins, imidazolidinedione and tetracycline groups. This finding is similar to another study in which treatment options for UTIs with or without ESBLs-producing Enterobacteriaceae include nitrofurantion, fosfomycin, fluoroquinolones, cefoxitin, piperacillin-tazobactam, carbapenems, ceftolozanetazobactam, ceftazidime-avibactam, and aminoglycosides. ${ }^{13}$ The same study also reported that fosfomycin, Ceftazidime-avibactam, polymyxin B, aztreonam, colistin, aminoglycosides, and tigecycline are treatment options for UTIs caused by carbapenem- resistant Enterobacteriaceae (CRE). ${ }^{13}$ Another study by Alamri et al also support the results of current study by reporting that fosfomycin, nitrofurantoin and pivmecillinam can be the first line management options for treating uncomplicated UTIs. ${ }^{17}$ Hence, ceftolozane/tazobactam are the approved antibacterial for empirical treatment of UTI and pyelonephritis in adults. ${ }^{17,18}$ Many relevant studies and trials have concluded that fosfomycin and Nitrofurantoin are still the magic bullets to treat any type of UTI. ${ }^{19,20}$ With Fosfomycin found to be the most reliable option for managing any type of UTI, whether complicated or non-complicated. ${ }^{14,21} \mathrm{~A}$ systematic literature review between 1946 to 2015 revealed that Nitrofurantoin harbors the properties of sterilizing urine and prevent the occurrence of UTI.22,23 However, the only point of concern regarding its prolonged use is severe gastrointestinal side effects. ${ }^{24}$ The results of current study showed that $94.1 \%$ cases of Enterococcus feacalis cases were found sensitive to tigecycline. The efficacy of tigecycline for UTI has not been extensively studied. Although a metaanalysis concluded that it is a good treatment option for multi-drug resistant (MDR) Gram-negative UTIs. ${ }^{25}$ Tigecycline is known to play a significant role in cases of UTI associated with polycystic kidney disease, impaired renal function and renal transplant. ${ }^{26}$

Our study showed least sensitivity for first-generation cephalosporins, tegicycline, teicoplanin, and Oxazolidinone (linezolid). This is in contrast to the published data which shows increased sensitivity of uropathogens to linezolid, daptomycin, and vancomycin. ${ }^{15}$ Similar findings were observed in another study which concluded that all Enterococci and Staphylococci harbor great sensitivity for linezolid, fosfomycin, vancomycin and teicoplanin. ${ }^{27}$ A Sri Lankan study reported that $95-100 \%$ ESBL producing organisms were sensitive to carbapenems, especially meropenem. Therefore, this group can also be used as first-line therapy for complicated UTIs. ${ }^{28}$ Moreover, the intravesical efficacy of gentamycin showed significant results in reducing the frequency of recurrent UTIs. ${ }^{29}$

The management options for ESBL producing organisms is biggest health challenge around the globe. ${ }^{30} \mathrm{~A}$ case series reported better outcomes for prophylactic use of a combination preparation, ceftibuten plus amoxicillin- 
clavulanic acid, ceftolozane/tazobactam and tegicycline even in ESBL producing organisms. ${ }^{31-33}$ Therefore, knowledge about the commonly prevalentorganisms and their susceptibility pattern can serve as an essential requirement for accurate management of urinary tract infections. ${ }^{34}$ Thus, the morbidity and mortality rates can be reduced due to proper management of complicated UTIs.

\section{Conclusion}

Carbapenems showed maximum sensitivity for all urine isolates. The second and third options were polymyxin $B$ and phosphonic acid derivatives, respectively.

\section{References}

1. Demilie $T$, Beyene G, Melaku S, Tsegaye W. Urinary bacterial profile and antibiotic susceptibility pattern among pregnant women in north west Ethiopia. Ethiop J Health Sci. 2012; 22(2): 121-28. PMID: 22876076

2. Parveen SS, Reddy SV, Rao RMV, Janardhan RR. Uropathogens and their drug susceptibility patterns among pregnant women in a teaching hospital. Ann Biol Res. 2011; 2(5): 516-21.

3. Ahmed E, Shahid HM, Ikhlaque SS, Ejaz M. Urinary tract bacterial pathogens and their sensitivity pattern. J Rawalpindi Med Coll. 2014; 18(2): 263-66.

4. Badr A, Shaikh AG. Recurrent urinary tract infections management in women: A review. Sultan QaboosUniv Med J. 2013; 13(3): 359-67.Doi: 10.12816/0003256

5. Pallett A, Hand K. Complicated urinary tract infections: practical solutions for the treatment of multiresistant Gram-negative bacteria. J Antimicrob Chemother. 2010; 65 (suppl 3): iii25-iii33. Doi: 10.1093/jac/dkq298

6. Kostakioti M, Hultgren SJ, Hadjfrangiskou M. Molecular blueprint of uropathogenic Escherichia coli virulence provides clues toward the development of anti-virulence therapy. Virulence. 2012; 3(7): 592-94. Doi: $10.4161 /$ viru. 22364

7. Foxman B. The epidemiology of urinary tract infection. Nat Rev Urol. 2010; 7(12): 653-60. Doi: 10.1038/nrurol.2010.190

8. Yousef SA. Clinical and laboratory profile of urinary tract infections associated with extended spectrum beta-lactamase producing Escherichia coli and
Klebsiella pneumoniae. Ann Clin Lab Sci. 2016; 46(4): 393-400.PMID: 27466299

9. Flores-Mireles AL, Walker JN, Caparon M, Hultgren SJ. Urinary tract infections: epidemiology, mechanisms of infection and treatment options. Nat Rev Microbiol. 2015; 13(5): 269-84. Doi: 10.1038/nrmicro3432

10. Ullah A, Shah SRH, Almugadam BS, Sadiqui S. Prevalence of symptomatic urinary tract infections and antimicrobial susceptibility patterns of isolated uropathogens in Kohat region of Pakistan. MOJ Biol Med. 2018; 3(4): 85-89.

11. George CE, Norman G, Ramana GV, Mukherjee D, Rao T. Treatment of uncomplicated symptomatic urinary tract infections: Resistance patterns and misuse of antibiotics. J Family Med Prim Care. 2015; 4(3): 416-421. Doi: 10.4103/2249-4863.161342

12. CLSI Performance Standards for Antimicrobial Susceptibility Testing. 27th ed. CLSI Supplement M100. Wayne, PA: Clinical Laboratory Standard Institute; $2017 . \quad$ Website: [https://clsi.org/media/1469/m100s27_sample.pdf] Retrieved on 7th Nov 2019.

13. Holm A, Cordoba G, Aabenhus R. Prescription of antibiotics for urinary tract infection in general practice in Denmark. Scand J Prim Health Care. 2019; 28: 1 7. Doi: $10.1080 / 02813432.2019 .1569425$.

14. Bader MS, Loeb M, Brooks AA. An update on the management of urinary tract infections in the era of antimicrobial resistance. Postgrad Med. 2017; 129(2): 242-58. Doi: 10.1080/00325481.2017.1246055.

15. Ou LB, Nadeau L. Fosfomycin susceptibility in multidrug-resistant Enterobacteriaceae species and Vancomycin-Resistant Enterococci urinary isolates. Can J Hosp Pharm. 2017; 70(5): 368-74. Doi: 10.4212/chp.v70i5.1698

16. Concia E, Bragantini D, Mazzaferri F. Clinical evaluation of guidelines and therapeutic approaches in multi drug-resistant urinary tract infections. J Chemother. 2017; 29(sup 1): 19-28. Doi: 10.1080/1120009X.2017.1380397.

17. Alamri A, Hamid ME, Abid M, Alwahhabi AM, Alqahtani KM, Alqarni MS, etal. Trend analysis of bacterial uropathogens and their susceptibility pattern: A 4-year (2013-2016) study from Aseer region, Saudi Arabia. Urol Ann. 2018; 10(1): 41-6. Doi: 10.4103/UA.UA_68_17. 
18. Scott LJ1. Ceftolozane/Tazobactam: A Review in Complicated Intra-Abdominal and Urinary Tract Infections. Drugs. 2016; 76(2): 231-42. Doi: 10.1007/s40265-015-0524-5.

19. Popejoy MW, Long J, Huntington JA. Analysis of patients with diabetes and complicated intraabdominal infection or complicated urinary tract infection in phase 3 trials of ceftolozane/tazobactam. BMC Infect Dis. 2017; 17(1): 316. Doi: 10.1186/s12879-017-2414-9.

20. Doesschate T, Mens SP, Nieuwkoop C, Geerlings SE, Hoepelman AIM, Bonten MJM, et al. Oral fosfomycin versus ciprofloxacin in women with $\mathrm{E}$. coli febrile urinary tract infection, a double-blind placebocontrolled randomized controlled non-inferiority trial (FORECAST). BMC Infect Dis. 2018; 18(1): 626. Doi: 10.1186/s12879-018-3562-2.

21. Jadoon SA, Ahmed A, IrshadR.SpectrumOf Bacterial Culture and Drug Sensitivity Vs Resistance in Uncomplicated Urinary Tract Infection. J Ayub Med Coll Abbottabad. 2018; 30(3): 432-38. PMID: 30465381

22. Keating GM. Fosfomycin trometamol: a review of its use as a single-dose oral treatment for patients with acute lower urinary tract infections and pregnant women with asymptomatic bacteriuria. Drugs. 2013; 73(17): 1951-66. Doi: 10.1007/s40265-013-0143-y.

23. Muller AE, Verhaegh EM, Harbarth S, Mouton JW, Huttner A. Nitrofurantoin's efficacy and safety as prophylaxis for urinary tract infections: a systematic review of the literature and meta-analysis of controlled trials. Clin Microbiol Infect. 2017; 23(6): 355-62. Doi: 10.1016/j.cmi.2016.08.003.

24. Fallah F, Yousefi M, Pourmand MR, Hashemi A, Alam A, Afshar D, etal. Phenotypic and genotypic study of biofilm formation in Enterococci isolated from urinary tract infections. Microb Pathog. 2017; 108: 85-90. Doi: 10.1016/j.micpath.2017.05.014.

25. Price JR, Guran LA, Gregory WT, Donagh MS. Nitrofurantoin vs other prophylactic agents in reducing recurrent urinary tract infections in adult women: a systematic review and meta-analysis. Am J Obstet Gynecol. 2016; 215(5): 548-60. Doi: 10.1016/j.ajog.2016.07.040.

26. Brust K, Evans A, Plemmons R. Tigecycline in treatment of multidrug-resistant Gram-negative bacillus urinary tract infections: a systematic review. J Antimicrob Chemother. 2014; 69(10): 2606-10. Doi: 10.1093/jac/dku189.
27. Yuan X, Liu T, Wu D, Wan Q. Epidemiology, susceptibility, and risk factors for acquisition of MDR/XDR Gram-negative bacteria among kidney transplant recipients with urinary tract infections. Infect Drug Resist. 2018; 11: 707-15. Doi: 10.2147/IDR.S163979. eCollection 2018.

28. Yang B, Yang F, Wang S, Wang Q, Liu Z, Feng W, et al. Analysis of the spectrum and antibiotic resistance of uropathogens in outpatients at a tertiary hospital. $\mathrm{J}$ Chemother. 2018; 30(3): 145-49. Doi: 10.1080/1120009X.2017.1418646.

29. Fernando MM, Luke WA, Miththinda JK, Wickramasinghe RD, Sebastiampillai BS, Gunathilake MP. Extended spectrum beta lactamase producing organisms causing urinary tract infections in Sri Lanka and their antibiotic susceptibility pattern -A hospital based cross sectional study. BMC Infect Dis. 2017; 17(1): 138. Doi: 10.1186/s12879-017-2250-y.

30. Seo YB, Lee J, Kim YK, Lee SS, Lee JA, Kim HY, et al. Randomized controlled trial of piperacillintazobactam, cefepime and ertapenem for the treatment of urinary tract infection caused by extended-spectrum beta-lactamase-producing Escherichia coli. BMC Infect Dis. 2017; 17(1): 404. Doi: 10.1186/s12879-017-2502-X.

31. Stuart CJ, Hall VM, Kortmann W, Verlind J, Mulder F, Scharringa $J$, et al. Ceftibuten plus amoxicillinclavulanic acid for oral treatment of urinary tract infections with ESBL producing $E$. coli and $K$. pneumoniae: a retrospective observational caseseries. Eur J Clin Microbiol Infect Dis. 2018; 37(10): 2021-25. Doi: 10.1007/s10096-018-3338-z.

32. Golan Y. Empiric therapy for hospital-acquired, Gramnegative complicated intra-abdominal infection and complicated urinary tract infections: a systematic literature review of current and emerging treatment options. BMC Infect Dis. 2015; 15: 313. Doi: 10.1186/s12879-015-1054-1.

33. Zhanel GG, Chung P, Adam H, Zelenitsky S, Denisuik A,Schweizer $F$, et al. Ceftolozane/tazobactam: a novel cephalosporin/ $\beta$-lactamase inhibitor combination with activity against multidrug-resistant gram-negative bacilli. Drugs. 2014; 74(1): 31-51. Doi: 10.1007/s40265-013-0168-2.

34. Alberici I, Bayazit AK, Drozdz D, Emre S, Fischbach $M$, Harambat J, et al. Pathogens causing urinary tract infections in infants: a European overview by the ESCAPE study group. Eur J Pediatr. 2015; 174(6): 783-90. Doi: 10.1007/s00431-014-2459-3 